Effects of preparation conditions on the optical properties of thin films of tellurium oxide

This article has been downloaded from IOPscience. Please scroll down to see the full text article.

2002 J. Phys. D: Appl. Phys. 35910

(http://iopscience.iop.org/0022-3727/35/9/312)

View the table of contents for this issue, or go to the journal homepage for more

Download details:

IP Address: 134.153.186.189

The article was downloaded on 01/03/2013 at 14:42

Please note that terms and conditions apply. 


\title{
Effects of preparation conditions on the optical properties of thin films of tellurium oxide
}

\author{
M F Al-Kuhaili ${ }^{1}$, S M A Durrani ${ }^{2}$, E E Khawaja ${ }^{2}$ and J Shirokoff ${ }^{3}$ \\ ${ }^{1}$ Physics Department, King Fahd University of Petroleum and Minerals, Dhahran 31261, \\ Saudi Arabia \\ ${ }^{2}$ Center for Applied Physical Sciences, Research Institute, King Fahd University of \\ Petroleum and Minerals, Dhahran 31261, Saudi Arabia \\ ${ }^{3}$ Faculty of Engineering and Applied Science, Memorial University of Newfoundland, \\ St John's, NF, Canada A1B3X5
}

Received 16 October 2001

Published 16 April 2002

Online at stacks.iop.org/JPhysD/35/910

\begin{abstract}
Thin films of tellurium oxide were prepared by thermal evaporation. The effects of preparation conditions and post-deposition vacuum annealing on the optical constants of the thin films were studied. Substantial changes in the optical constants, density, structure and stoichiometry were observed following changes in the preparation conditions and annealing. The majority of the films were found to be deficient in oxygen. The presence of metallic Te was detected in films deposited on heated substrates and in all the films that were annealed. All the samples showed some degree of absorption at photon energies below the band gap. One explanation for this absorption could be oxygen deficiency and the presence of metallic Te.
\end{abstract}

\section{Introduction}

Tellurium oxide is one of the most interesting materials for the realization of optical recording systems $[1,2]$, featuring low writing energies, high stability in a non-controlled atmosphere, high resolution and low fabrication cost [3]. All these properties make tellurium oxide thin films suitable as an optical disk active medium. The optical properties of thin films of tellurium oxide have been the subject of several studies $[1,3,4-8]$. In the literature, it is not unusual to observe a wide disparity in the reported values of the optical constants (refractive $(n)$ and absorption $(k)$ indices). Only the influence of the deposition conditions (i.e. substrate temperature, deposition rate, oxygen pressure) as well as the method of producing the film, can account for these widely divergent values $[1,3,4-8]$.

The optical properties of oxide films are dependent on chemical composition and microstructure. It is well known that oxide films prepared by physical vapour deposition techniques can be non-stoichiometric as the compound can decompose in the vapour phase and condense with a chemical composition differing from that of the source material. Therefore, careful measurement of the optical constants is of great importance.
In this work, tellurium oxide thin films were prepared by thermal evaporation. The aim of the work was to study the possible variations in the values of $n$ and $k$ as a result of changing the oxygen partial pressure and substrate temperature. Furthermore, the effects of post-deposition vacuum annealing were also investigated. Chemical and structural analyses of the films were performed using $\mathrm{x}$-ray photoelectron spectroscopy (XPS) and x-ray diffraction (XRD), respectively.

\section{Experimental}

Thin films of tellurium oxide of thickness $60-200 \mathrm{~nm}$ were prepared simultaneously on fused silica and glass substrates in a Leybold model L560 box coater pumped by a turbomolecular pump. The silica substrates were in the form of wedges of a $3^{\circ}$ angle. The coating chamber was pumped to a base pressure of $2 \times 10^{-6}$ mbar. The source material, $\mathrm{TeO}_{2}$, of purity $99.9995 \%$, was evaporated from a silica crucible heated by an external tungsten coil. The substrates were rotated during the deposition. The rate of evaporation used was in the range from 0.1 to $0.3 \mathrm{~nm} \mathrm{~s}^{-1}$. This was monitored using a quartz crystal thickness monitor. The source-to-substrate distance was $40 \mathrm{~cm}$. 
The deposition parameters varied in the present work were the substrate temperature and the oxygen partial pressure. In the preparation of some films, oxygen was readmitted to raise the pressure of the coating chamber. In the coating chamber, the substrates were either maintained at ambient temperature or were heated to a temperature of $300^{\circ} \mathrm{C}$. The settings used were: (a) films deposited on unheated substrates in the presence of oxygen, labelled as $\operatorname{US}(\mathrm{O})$ : US $(\mathrm{O} 1)$ when the oxygen partial pressure was $5 \times 10^{-4}$ mbar and US(O2) when it was $1 \times 10^{-3}$ mbar; (b) films deposited on unheated substrates without oxygen, US(WO); (c) films deposited on heated substrates with oxygen, $\mathrm{HS}(\mathrm{O} 1)$; and (d) films deposited on heated substrates without oxygen, HS(WO).

After the films were deposited, they were removed from the coating chamber and exposed to the ambient atmosphere, prior to measurement of optical properties, XPS and XRD. Measurements were also carried out on the films that were annealed in vacuum (pressure of about $6 \times 10^{-6} \mathrm{mbar}$ ) at a temperature of $300^{\circ} \mathrm{C}$ for $4 \mathrm{~h}$. For the annealed films, the above labelling with the addition of a letter A was used; for example, US(WO)A represents an annealed film of the type US(WO).

The normal incidence transmittance of the films on fused silica wedges was measured over the $250-700 \mathrm{~nm}$ wavelength range using a double-beam Bausch and Lomb spectrophotometer, model Spectronic 2000. The use of a wedge substrate ensured that reflections from the back of the substrate were deflected out of the optical path of the instrument so that multiple reflections in the substrate did not affect the measurements. However, the wedge angle was small enough for transmission across the back face to be given accurately enough by the transmittance formula for normal incidence.

XPS was performed in a VG Scientific ESCALAB MKII spectrometer equipped with a dual aluminium-magnesium anode. The system has been described elsewhere [10].

Films deposited on glass substrates were used for XRD analysis. The samples were mounted in a Rigaku Ru-200 x-ray diffractometer with the film side exposed to the x-ray source. The diffractometer is equipped with a $\mathrm{Cu}$ rotating anode and a monochromator for sample irradiation and detection of the $\mathrm{Cu} \mathrm{K} \alpha$ radiation scattered from the sample surface. An identification search match of the various phases present in the samples was performed using the MDI Jade software [11]. Crystallite size measurements were performed on XRD data using the MDI Jade software which calculates the crystallite size from line broadening measurements using the Scherrer formula convoluted to that of the peak (line) full width at half maximum (FWHM). The mean crystallite size results must lie between about 2-300 $\mathrm{nm}$ to be acceptable when using the Scherrer formula [12].

\section{Results and discussion}

A method for the determination of the refractive $(n)$ and absorption $(k)$ indices of thin dielectric films on transparent substrates was reported earlier [9]. It requires measurement at normal incidence of the transmittance from two films of a given dielectric of different thickness as a function of wavelength $(\lambda)$. The method has been applied successfully to films of different dielectrics $[13,14]$. The results ( $n$ and $k$ ) discussed below were obtained by the use of this method. The results for different specimens (sets of two films of different thickness) of a given type of film were reproducible to within $2 \%$ in the values of $n$, and within $5 \%$ in the values of $k$.

Average dispersion $(n-\lambda)$ and absorption $(k-\lambda)$ curves for different types of films are shown in figures 1 and 2, respectively. For comparison, the values of the refractive indices (for ordinary $\left(n_{\mathrm{o}}\right)$ and extraordinary $\left(n_{\mathrm{e}}\right)$ rays) obtained from single-crystal paratellurite $\left(\mathrm{TeO}_{2}\right)$ [15] are reproduced in figure 1. Generally, the density of a film is lower than that of the corresponding bulk material. Therefore, from the LorentzLorenz law, the refractive index of a film should be lower than that of the corresponding bulk material. Indeed, this was the case as can be seen in figure 1. For the films deposited on

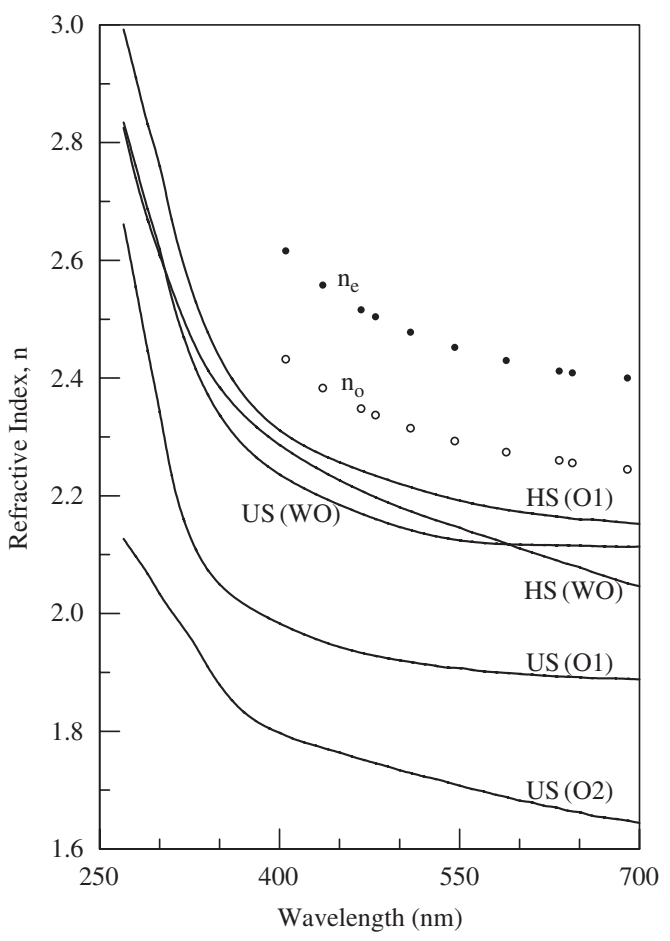

Figure 1. Refractive index versus wavelength curves for different types of films. For comparison, refractive indices $n_{\mathrm{o}}$ (ordinary ray) and $n_{\mathrm{e}}$ (extraordinary ray) measured for single-crystal paratellurite [15] are shown.

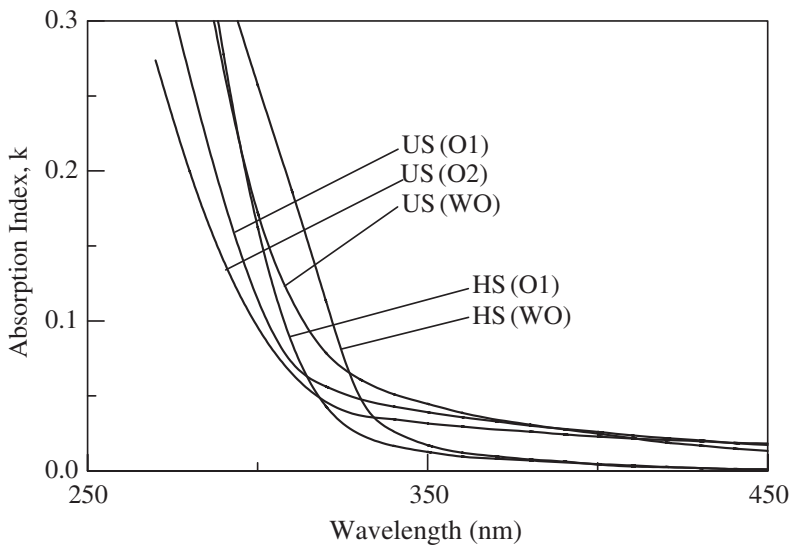

Figure 2. Absorption index versus wavelength curves for different types of films. 
unheated substrates, both $n$ (figure 1) and $k$ (figure 2) decreased with increasing oxygen partial pressure. Similar changes of the indices have been previously observed in films of some other dielectrics [16-18]. These have been attributed to a decrease in the density of a film upon an increase in the oxygen partial pressure. Present measurements are insufficient to yield an accurate value of the density of the film. However, the densities of the films relative to each other can be estimated as follows.

In an evaporation chamber, the amounts of mass per unit area (density $(\rho) \times$ thickness $(d)$ of a film) received by a rotating substrate $\left(\rho_{\mathrm{f}} d_{\mathrm{f}}\right)$ and a stationary quartz crystal $\left(\rho_{\mathrm{qc}} d_{\mathrm{qc}}\right)$ are related to each other by $\rho_{\mathrm{f}} d_{\mathrm{f}}=G \rho_{\mathrm{qc}} d_{\mathrm{qc}}$, where $\rho_{\mathrm{f}}$ is the density of the film and $\rho_{\mathrm{qc}}$ is the density of the material to be evaporated. The factor $G$ takes into account the influence of the difference in position between the crystal and the substrate, assuming that the substrate and the crystal are at the same temperature. In the present situation, the average distance of the rotating substrate from the evaporation source is larger than the fixed distance between the crystal and the evaporation source. Thus, it is expected that the mass per unit area received by the substrate is smaller than that received by the crystal. This means that the factor $G$ is less than one. In fact, in an earlier study, $G$ was estimated to be 0.62 [13]. For films deposited on unheated substrates, the temperatures of the substrate and the crystal are the same; therefore, $G$ is a constant. Thus, $\rho_{\mathrm{f}} \propto d_{\mathrm{qc}} / d_{\mathrm{f}}$, where $d_{\mathrm{qc}}$ is the thickness as fed into the quartz crystal thickness monitor and $d_{\mathrm{f}}$ is the film thickness as measured by the optical method [9]. These measurements produced a ratio of 1.39 for US(WO), 1.25 for $\mathrm{US}(\mathrm{O} 1)$ and 1.19 for $\mathrm{US}(\mathrm{O} 2)$ film types. These values suggest that the density of the films deposited on unheated substrates decreases as the partial pressure of oxygen is increased. Films deposited on heated substrates, i.e. HS(WO) and HS(O1) types, had refractive indices higher than those for the films deposited on unheated substrates (figure 1). Similar results were obtained for tungsten oxide films $[16,17]$.

In previous studies, the films were prepared under different conditions and, as can be seen in figures 1 and 2, these have substantial effects on the $n$ and $k$ values. Reported values of $n$ measured around $\lambda=800 \mathrm{~nm}$ fall in the range 1.8-2.26 $[2,4,6,7]$.

The optical absorption in the UV (figure 2) is dominated by the band gap $\left(E_{\mathrm{g}}\right)$ of the semiconductor. The band gap is wide enough to render the films transparent. The value of $E_{\mathrm{g}}$ can be derived by applying the relation

$$
\alpha h v=B\left(h v-E_{\mathrm{g}}\right)^{\eta}
$$

where $\alpha(=4 \pi k / \lambda)$ is the absorption coefficient at photon energy $h v, B$ is a constant and $\eta$ depends on the kind of optical transitions that prevail. Generally, for amorphous films, the above relationship is well obeyed, provided that $\eta=2$. Figure 3 shows a plot of $(\alpha h v)^{1 / 2}$ versus $h v$ for films of the US(WO) type. It can be seen that two distinct linear relations exist, corresponding to different absorption processes. Similar plots were obtained for the other types of films. The intercepts of the two lines with the photon energy axis $\left(E_{\mathrm{g} 1}\right.$ and $E_{\mathrm{g} 2}$, figure 3) yield the onsets for the two different absorption processes. The gap $E_{\mathrm{g} 1}$ was found to be about $3.65 \mathrm{eV}$ and remained almost constant (within 2\%) for all types of films.

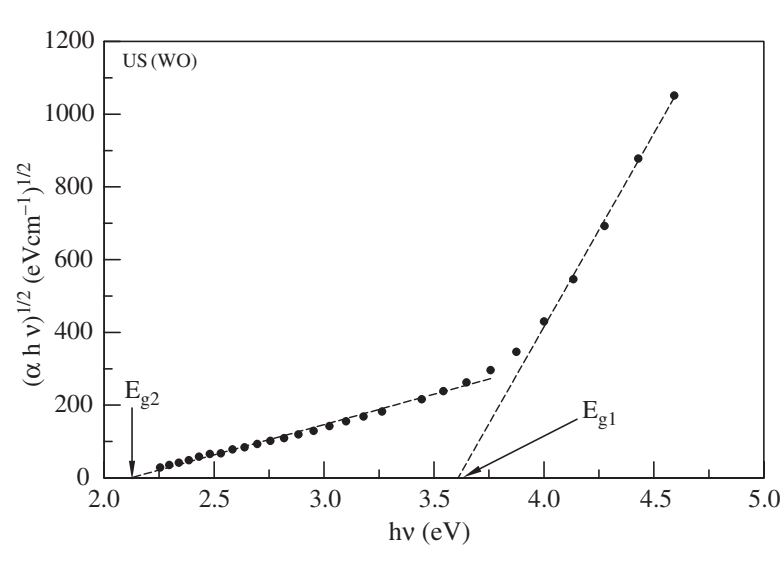

Figure 3. Plot of $(\alpha h v)^{1 / 2}$ versus $h v$ for film type US(WO).

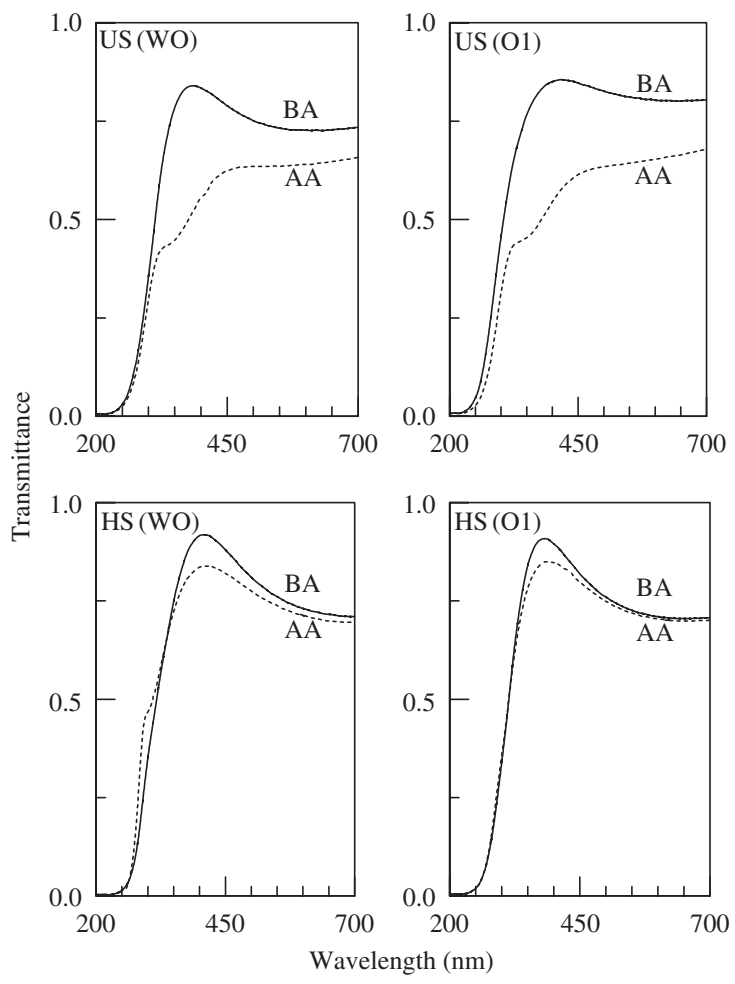

Figure 4. Transmittance of the films before (BA) and after annealing (AA).

On the other hand, the gap $E_{\mathrm{g} 2}$ at $2.12 \mathrm{eV}$ (figure 3) for the US(WO) type shifted to lower energies down to $1.25 \mathrm{eV}$ in the case of films of the US(O) type and to higher energies up to $2.59 \mathrm{eV}$ in the $\mathrm{HS}(\mathrm{WO})$ and $\mathrm{HS}(\mathrm{O})$ types. The reported values of the band gap for tellurium oxide films lie in the range $3.5-3.75 \mathrm{eV}[5,7]$. It is suggested that $E_{\mathrm{g} 1}$ may represent the onset of intrinsic absorption of the oxide while $E_{\mathrm{g} 2}$ may represent the onset of absorption involving some defect states. The defect states may change with the type of film, and thus result in different values of the onset for different types of films.

Transmittance measurements made before and after annealing of different types of films are presented in figure 4. The films were annealed in vacuum (pressure $\sim 8 \times 10^{-6} \mathrm{mbar}$ ) at a temperature of $300^{\circ} \mathrm{C}$ for $4 \mathrm{~h}$. The optical constants of the films obtained before (BA) and after (AA) annealing are shown in figure 5 for the US(WO) type and figure 6 for 
Optical properties of thin films of tellurium oxide
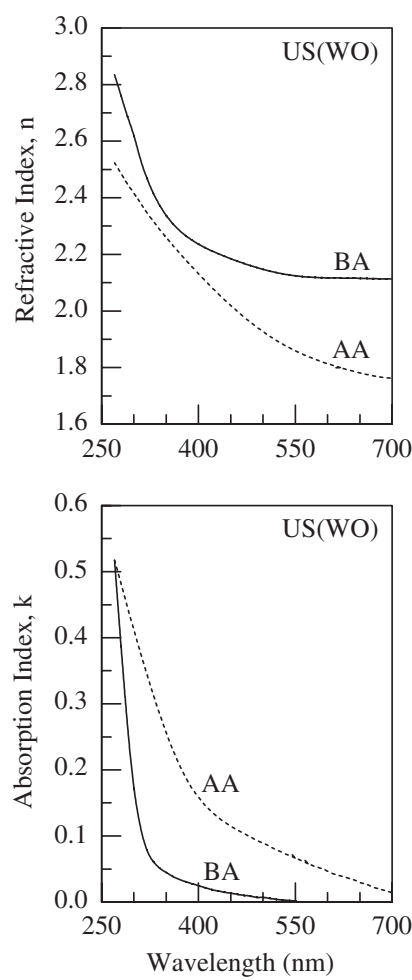

Figure 5. Refractive and absorption indices of the sample US(WO) before (BA) and after annealing (AA).
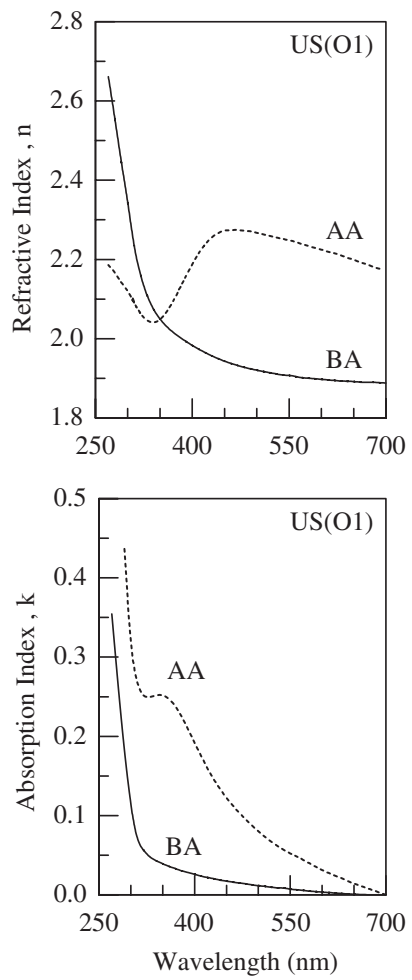

Figure 6. Refractive and absorption indices of the sample US(O1) before (BA) and after annealing (AA).

the US(O1) type. These substantial changes in the optical constants following annealing of the films can be attributed to changes in the stoichiometry and structure of the films, as will be discussed later.

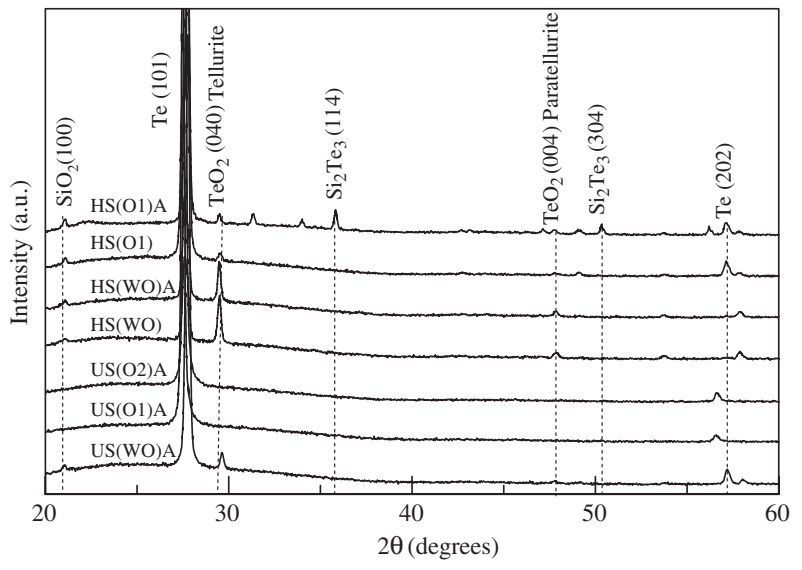

Figure 7. XRD patterns of different types of films.

Table 1. Mean crystallite size of crystallites of (a) metallic Te, (b) $\mathrm{TeO}_{2}$ tellurite and (c) $\mathrm{TeO}_{2}$ paratellurite

\begin{tabular}{llll}
\hline Film type & $\begin{array}{l}\text { Metallic } \\
(\mathrm{nm})\end{array}$ & $\begin{array}{l}\mathrm{TeO}_{2} \text { tellurite } \\
(\mathrm{nm})\end{array}$ & $\begin{array}{l}\mathrm{TeO}_{2} \text { paratellurite } \\
(\mathrm{nm})\end{array}$ \\
\hline US(WO) & $\mathrm{ND}$ & $\mathrm{ND}$ & $\mathrm{ND}$ \\
$\mathrm{US}($ WO)A & 39.2 & 27.3 & $\mathrm{ND}$ \\
$\mathrm{US}(\mathrm{O} 1)$ & $\mathrm{ND}$ & $\mathrm{ND}$ & $\mathrm{ND}$ \\
$\mathrm{US}(\mathrm{O} 1) \mathrm{A}$ & 50.1 & $\mathrm{ND}$ & $\mathrm{ND}$ \\
$\mathrm{US}(\mathrm{O} 2)$ & $\mathrm{ND}$ & $\mathrm{ND}$ & $\mathrm{ND}$ \\
$\mathrm{US}(\mathrm{O} 2) \mathrm{A}$ & 52.0 & $\mathrm{ND}$ & $\mathrm{ND}$ \\
$\mathrm{HS}($ WO) & 29.9 & 44.1 & 15.5 \\
$\mathrm{HS}($ WO)A & 26.5 & 52.6 & 21.1 \\
HS(O1) & 26.3 & 34.0 & $\mathrm{ND}$ \\
HS(O1)A & 36.0 & 49.5 & $\mathrm{ND}$ \\
\hline
\end{tabular}

ND: not detected.

The accuracy of the mean crystallite size measurements was about $5-10 \%$.

The XRD data for the as-deposited films on unheated substrates indicated that these films were amorphous. On the other hand, as shown in figure 7, diffraction lines were observed in films of the US(WO)A, US(O)A, HS(WO), HS(WO)A, $\mathrm{HS}(\mathrm{O})$ and $\mathrm{HS}(\mathrm{O}) \mathrm{A}$ types. As marked in figure 7 , lines corresponding to crystallites of metallic $\mathrm{Te}, \mathrm{TeO}_{2}$ tellurite and $\mathrm{TeO}_{2}$ paratellurite were detected. The presence of lines of crystallites such as $\mathrm{SiO}_{2}$ and $\mathrm{Si}_{2} \mathrm{Te}_{3}$ was also detected in some of the films (see figure 7). Crystallite sizes determined from line broadening measurements are listed in table 1 . The lines used were $\mathrm{Te}(101), \mathrm{TeO}_{2}$ tellurite $(040)$ and $\mathrm{TeO}_{2}$ paratellurite (004). The presence of metallic Te in tellurium oxide films has been the subject of a number of investigations, which have confirmed the ability of this material to exhibit appropriate changes in its optical properties following thermal treatment $[1-4,8]$. These changes have been attributed to the observed crystallization of Te micrograins in the films upon thermal treatment [2]. Overall, XRD studies on heat-treated tellurium oxide films [18] have revealed that the films are initially amorphous prior to heat treatment, followed by the formation of crystalline Te metal with subsequent crystallization of $\mathrm{TeO}_{2}$ at higher temperatures.

Figures 8 and 9 show the XPS spectra of the Te $3 \mathrm{~d}_{5 / 2}$ and O 1s peaks, respectively, for a film of the US(O1) type. Similar spectra were obtained for the other types of films. Nearly independent of the type of film, the values of the FWHM of the 


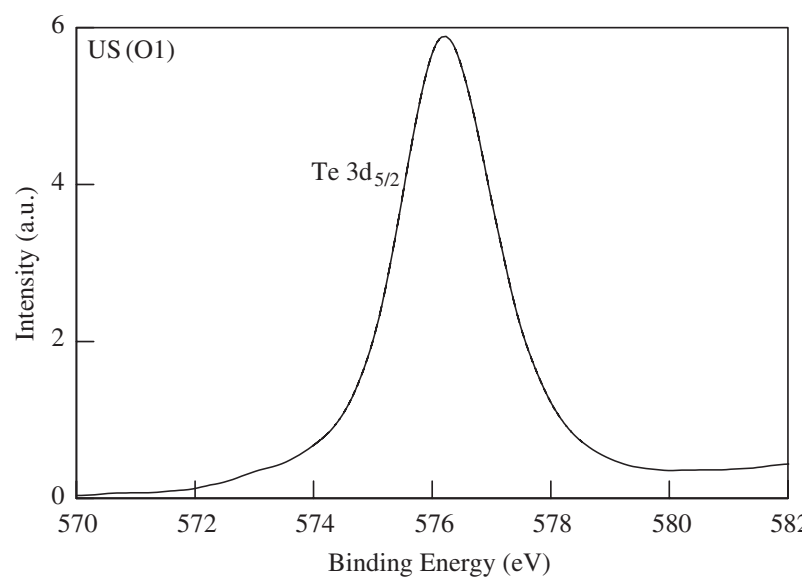

Figure 8. XPS spectrum of Te $3 d_{5 / 2}$ of film type US(O1).

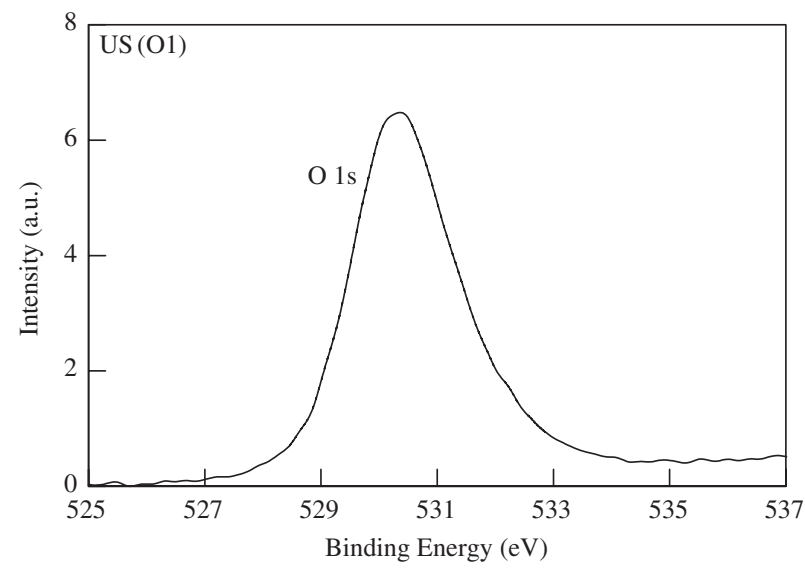

Figure 9. XPS spectrum of O 1s of film type US(O1).

Table 2. Summary of XPS results.

\begin{tabular}{llll}
\hline & $\begin{array}{l}\text { Peak position } \\
\text { O 1s } \\
(\mathrm{eV})\end{array}$ & $\begin{array}{l}\text { Peak position } \\
\text { Te 3d } 5 / 2 \\
(\mathrm{eV})\end{array}$ & $\begin{array}{l}\text { Atomic ratio } \\
\text { O : Te }\end{array}$ \\
\hline US(WO) & 529.8 & 575.7 & 1.85 \\
US(WO)A & 529.9 & 575.8 & 1.81 \\
US(O1) & 530.3 & 576.2 & 2.02 \\
US(O1)A & 530.1 & 575.9 & 1.83 \\
US(O2) & 530.3 & 576.2 & 2.06 \\
US(O2)A & 530.3 & 576.1 & 1.95 \\
HS(WO) & 529.7 & 575.6 & 1.84 \\
HS(WO)A & 529.9 & 575.8 & 1.81 \\
HS(O1) & 530.2 & 576.0 & 1.95 \\
HS(O1)A & 530.3 & 576.1 & 1.85 \\
\hline
\end{tabular}

All peaks are referenced to $\mathrm{C} 1 \mathrm{~s}$ at $284.6 \mathrm{eV}$. The energy resolution of the system is about $1.0 \mathrm{eV}$.

Te $3 \mathrm{~d}_{5 / 2}$ and $\mathrm{O} 1 \mathrm{~s}$ peaks were found to be $1.86 \mathrm{eV}$ (within 2\%) and $1.95 \mathrm{eV}$ (within 5\%), respectively. The energy positions of the two peaks for the different types of films are given in table 2 . The atomic ratio of $\mathrm{O}: \mathrm{Te}$ obtained from the areas of the two peaks (figures 8 and 9) is also listed in table 2. The values of the ratio may be somewhat uncertain because of the presence of some absorbed gases on the surface of the samples, which may contain oxygen, air and water vapour [19]. In fact, it was reported [20] that the ratio measured by XPS (surface analysis) is systematically about 0.5 units higher than the corresponding value obtained by nuclear reaction analysis and Rutherford backscattering spectrometry (bulk analysis) techniques. In the present work, therefore, it is suggested that the films have a stoichiometry of $\mathrm{TeO}_{x}$, where $x$ may be less than 2.0. An $x$ value of less than 2.0 is further supported by the presence of (a) metallic Te detected by XRD (figure 7) and (b) absorption in the films (figure 2) in the wavelength region higher than $350 \mathrm{~nm}$, i.e. for photon energies below the band gap. In earlier studies [2,3] on $\mathrm{TeO}_{x}$ films with $x$ less than 1.1, the XPS revealed two well-separated peaks $\left(\mathrm{Te} 3 \mathrm{~d}_{5 / 2}\right)$ of nearly the same amplitude, attributable to Te metal $(\sim 573 \mathrm{eV})$ and $\mathrm{Te}$ in $\mathrm{TeO}_{2}$ $(\sim 576 \mathrm{eV})$, respectively. However, in the present work, the Te $3 \mathrm{~d}_{5 / 2}$ spectrum (figure 8 ) looks like a single peak centred at about $576 \mathrm{eV}$. Therefore, it is suggested that the value of $x$ may be closer to 2.0 rather than 1.1, i.e. the content of metallic Te in the films may be very small. The exact content of metallic $\mathrm{Te}$ in the films may not be easily determined, although, in the following, a rough estimate of this is made. A deconvolution method in which the Te $3 \mathrm{~d}_{5 / 2}$ spectrum is assumed to be composed of two overlapping peaks (corresponding to Te and $\mathrm{TeO}_{2}$ ) was undertaken. Each component peak in the spectrum was fitted to a sum of weighted Lorentzian-Gaussian peaks with a linear sloping background by means of a least-squares fitting program [21]. A best fit to the experimental data was found by varying the peak position, width and intensity of each of the two component peaks. Using the values of the areas under the two peaks, the atomic ratio of metallic Te : total Te for each type of film was determined. There were large variations in the values of this ratio from one type of film to another, but it was found that the metallic Te was always less than $2.3 \%$ of the total Te in a sample.

Annealing a film in vacuum leads to (a) a decrease in its transmittance (figure 4), (b) loss of the oxygen content of the film (table 2), both these phenomena being common to films of other metal oxides $[17,18]$, and (c) formation of nanocrystallites (table 1). Moreover, upon annealing, the thickness of the film was decreased by about $25 \%$ in the case of the US(O1) type, while it increased by about the same percentage in the US(WO) type. A decrease in the thickness of a sample results in an increase in its density that, in turn, leads to a higher refractive index (Lorentz-Lorenz law). The opposite is true when the thickness of a sample increases. Indeed, this is the case, as shown in figures 5 and 6. However, the increase in absorption at the longer wavelength side of the spectra, following annealing, observed for both types (figures 5 and 6) may be due to the formation of defects, as discussed below.

The absorption curve for the US(O1)A type plotted in figure 6 shows the development of an absorption band (appearing as a shoulder). The peak of this band appears to be at a photon energy just below the band edge $E_{\mathrm{g} 1} \sim 3.65 \mathrm{eV}$. All the other types of films do have some absorption in the energy region below the band gap, but these are much weaker than the band observed for the US(O1)A type. In an earlier study [7] on tellurium oxide films, an absorption band centred around $360 \mathrm{~nm}$, similar to the one seen in figure 6, was observed for the films that were reduced in cracked ammonia at $350^{\circ} \mathrm{C}$, while as-grown films did not show such a band. The defects giving rise to this absorption band were attributed to the $\mathrm{F}$ centres 
caused by oxygen deficiency [7]. A value of the atomic ratio $\mathrm{O}:$ Te of less than 2 indicates a deficiency in oxygen. The smaller the value of the ratio, the larger the deficiency. It is clear from table 2 that there are some other types that have a higher deficiency of oxygen than the US(O1)A type. Thus, the defects caused by oxygen deficiency alone cannot justify the existence of the band in figure 6. (This statement may be treated with some caution, as XPS is a surface analysis technique, and therefore the results obtained by this technique may not represent the bulk of the film material.) A notable difference between the US(O1)A or US(O2)A type and any of the remaining types from table 1 is the relatively large size of the metallic Te crystallites in the US(O1)A type. It is known that metal oxide stoichiometry can be changed very easily at high temperatures by exposure to a vacuum [22]. When a crystal made non-stoichiometrically by such a procedure is rapidly cooled, a large flaw (defect) density can be quenched in. Such quenched-in excess defects are liable to precipitate on grain boundaries or on dislocations, where they exist [22]. Therefore, it is suggested that in the US(O1)A type, the large size of Te crystallites and their larger boundaries may have a larger density of defects than the other types of films. Absorption is expected to increase with the density of defects in a sample.

It is interesting to note that in a similar investigation carried out earlier on films of tungsten oxide [17], an absorption band at photon energies much lower than the band gap energy was detected in the US(O)A type of films. Such a band was not present in pure $\mathrm{WO}_{3}$ films and, in all other types of films, absorption in the region of this band was extremely weak. In fact, this band in the US(O)A type closely matched an absorption band developed on the colouration of tungsten oxide films by electrochromic means. It was recently observed that annealing of tungsten oxide films in $\mathrm{UHV}$ at $300^{\circ} \mathrm{C}$ produced an increase of metallic states at the Fermi level [23].

\section{Conclusions}

The optical constants of thin films of tellurium oxide prepared under different conditions have been determined. It was found that the optical constants of a film depend critically on the substrate temperature and the oxygen partial pressure during evaporation. The presence of absorption in the photon energy region below the band gap, and to some extent the XPS results, suggested that the films were non-stoichiometric (deficient in oxygen). The presence of metallic Te in films deposited on heated substrates and all the annealed films was detected by XRD. All the samples showed some degree of absorption at photon energies below the band gap. It is suggested that this absorption may arise from defect centres, the density of which increases with the size of the Te metal nanocrystallites.

\section{Acknowledgments}

The support provided by the Research Institute and the Physics Department of King Fahd University of Petroleum and Minerals is acknowledged. Thanks are due to Dr N Tabet for his assistance with XPS measurements. J S would like to thank the Natural Sciences and Engineering Research Council of Canada for financial support.

\section{References}

[1] Ohta T, Takenga M, Akahira N and Yamashita T 1982 J. Appl. Phys. $\mathbf{5 3} 8497$

[2] Takenaga M, Yamada N, Nishiuchi K, Akahira N, Ohta T, Nakamura S and Yamashita T 1983 J. Appl. Phys. 545376

[3] Lee Wen-Yaung 1985 J. Vac. Sci. Technol. A 3640

[4] Seki H 1983 Appl. Phys. Lett. 431000

[5] Al-Ani S K J and Hogarth C A 1985 Int. J. Electron. 58123

[6] Lakshminarayan N, Radhakrishnan M and Balasubraman C 1986 J. Mater. Sci. 21246

[7] Mansingh A and Kumar S 1988 Thin Solid Films 161101

[8] Di Giulio M, Nicotra M C, Re M, Rella R and Siciliano P 1993 Appl. Surf. Sci. 65/66 313

[9] Khawaja E E and Bouamrane F 1993 Appl. Opt. 321168

[10] Khawaja E E, Hussain Z, Jazzar M S and Dabbousi O B 1987 J. Non-Cryst. Solids 9345

[11] MDI Jade Software 1990 Materials Data Inc., Livermore, CA

[12] Barrett C S and Massalski T B 1966 Structure of Metals 3rd edn (New York: McGraw-Hill) p 155

[13] Khawaja E E, Bouamrane F, Al-Adel F F, Hallak A B, Daous M A and Salim M A 1994 Thin Solid Films 240121

[14] Khawaja E E, Durrani S M A and Daous M A 1997 J. Phys.: Condens. Matter 99381

[15] Uchida N 1971 Phys. Rev. B 43736

[16] Bange K 1999 Solar Energy Mater. Solar Cells 58

[17] Durrani S M A, Khawaja E E, Salim M A, Al-Kuhaili M F and Al-Shukri A M 2002 Solar Energy Mater. Solar Cells 71313

[18] Hodgson S N B and Weng L 2000 J. Sol-Gel. Sci. Technol. 18145

[19] Khawaja E E, Bouamrane F, Hallak A B, Daous M A and Salim M A 1993 J. Vac. Sci. Technol. A 11580

[20] Di Giulio M, Rella R, Siciliano P and Cucurachi S 1992 Vacuum $\mathbf{4 3} 305$

[21] Proctor A and Sherwood P M A 1980 Anal. Chem. 52660

[22] Blakemore J S 1985 Solid State Physics 2nd edn (Cambridge: Cambridge University Press) p 78

[23] Santucci S, Lozzi L, Maccallini E, Passacantando M, Ottaviano L and Cantalini C 2001 J. Vac. Sci. Technol. A 19 1467 